\title{
EVALUATING THE TOUGHNESS OF PIPE STEELS BY IMPACT FRACTURE CURVES
}

\author{
V. A. Khotinov*, V. M. Farber, A. N. Morozova \\ ${ }^{1}$ Ural Federal University, 19 Mira st., Ekaterinburg, Russia Federation, 620002 \\ * Corresponding author. E-mail: khotinov@yandex.ru ; address for correspondence 620002, ul. Mira 19 Ekaterinburg, \\ Russia Federation. Tel.: +79089019912; fax:+7 (343) 375-44-39
}

To study the correlation of instrumented Charpy load-displacement curves with zones on the fracture surface, impact V-notched tests on low- and medium-carbon pipeline steels have been performed. New parameters of smoothed instrumented impact load vs displacement curves are proposed for impact properties characterization. The co-analysis of the instrumented impact test curves and the fracture surface zones allows new impact parameters for fracture characterization to be obtained in addition to standard required characteristics (impact toughness, transition temperature, impact yield strength etc.).

Keywords: instrumented Charpy test, pipeline steels, fracture surface, crack propagation stages, stress oscillations.

\section{References}

1. Gudkov A.A. Treschinosoikost stali. [Crack Resistance of Steels]. Moscow, Metallurgia Publ., 1989. 376 p. (In Russian).

2. Makhutov M.A., Permyakov V.B., Botvina L.R., Kravtsova Yu.A. A complex analysis of the mechanical properties the material made to substantiate the lifetime and safety of products. Problemi bezopasnosti i chrezvichainikh situatsiy, 2006, no. 3, pp. 65-76. (In Russian).

3. Botvina L.R. Razrushenie: kinetika, mekhanizmi, obshie zakonomernosti [Rfacture: Kinetics, Mechanisms, General Laws]. Moscow, Nauka Publ., 2008, 334 p. (In Russian).

4. ASTM E2298. Standard test method for instrumented impact testing of metallic materials. ASTM Publ., 2013. 9 p.

5. Manahan M.P., Stonesifer R.B. The difference between total absorbed energy measured using an instrumented striker and that obtained using an optical decoder. In book «Pendulum Impact Testing: A Century of Progress», ESIS STP 1380, 2000, pp.181-197.

6. Tronskar J.P., Mannan M.A., Lai M.O. Measurements of fracture initiation toughness and crack resistance in instrumented Charpy impact testing. Eng. Fract. Mech., 2002, vol. 69, pp. 321-338.

7. Viehrig H.-W., Boehmert J., Richter H., Valo M. Use of instrumented Charpy test for determination of crack initiation toughness. In book «Pendulum Impact Testing: A Century of Progress», ESIS STP 1380. 2000, pp. 354-365.

8. Arabey A.B., Farber V.M., Pyshmintsev I.Yu. et al. The microstructure and dispersed phases in the high-strength steels of large gas pipes. Nauka I tekhnika v gazovoi promishlennosti, 2011 , no. 4, pp. 86-91. (In Russian).

9. Tykhontseva N.T., Gorozhanin P.Yu., Zhukova S.Yu. et al. Searching the compositions and heat treatment conditions for high-strength tubing. Stal, 2006, no. 8, pp. 70-73. (In Russian).

10. Farber V.M., Arabey A.B., Pyshmintsev I.Yu., Khotinov V.A. et al. The fractographic diagnostics of the crack resistance of X80 (K65) pipes by toughness testing results. Trudy XVIII Int. conf. «Trubi-2010» [Proceedings of the $18^{\text {th }}$ Int. Conf. "Pipes-2010"]. Chelyabinsk : RosNITI Publ., 2010, pp. 108-117. (In Russian).

11. Farber V.M., Selivanova O.V. Classifying the processes of stress relaxation and their manifestation during plastic deformation of metals. Metally, 2001, no. 1, pp. 110-114. (In Russian). 
12. Kan R.U., Haazen P. Phyzicheskoe metallovedenie. T. 3 [Physical Metallurgy. Vol. 3]. Moscow, Metallurgia Publ., 1987. 664 p. (In Russian).

13. Arabey A.B., Pyshmintsev I.Yu., Farber V.M., Khotinov V.A. et al. Peculiarities of the fracture of X80 (K65) pipe steels. Izvestia vuzov. Chernaya metallurgia, 2012, no. 3, pp. 12-20. (In Russian). 
УДК 621.774.35:621.789

\title{
ОЦЕНКА УРОВНЯ ВЯЗКОСТИ ТРУБНЫХ СТАЛЕЙ С ПОМОЩЬЮ ДИАГРАММ УДАРНОГО РАЗРУШЕНИЯ
}

\author{
В. А. Хотинов*, В. М. Фарбер, А. Н. Морозова \\ ФГАОУ ВПО «Уральский федеральный университет имени первого Президента России Б. Н. Ельцина», \\ ул. Мира 19, Екатеринбург, Российская Федераџия \\ *Ответственный автор. Электронная почта: khotinov@,yandex.ru; адрес для переписки: 620002 ул. Мира 19, \\ Екатеринбург, Российская Федерация. Телефон: +79089019912; факс:+7 (343) 375-44-39
}

Проведен анализ диаграмм ударного разрушения образцов Шарпи низко- и среднеуглеродистых сталей, построенных в координатах усилие - прогиб образца. Установлено, что для вязкого состояния характерно осциллирование кривой усилия на диаграмме. Определены характерные параметры и участки диаграмм, соответствующие различным механизмам разрушения. С привлечением фрактографического анализа выявлена связь отдельных участков диаграммы разрушения с определенными зонами на поверхности излома образцов.

Ключевые слова: диаграммы ударного разрушения, поверхность разрушения, стадии разрушения, осциллляция нагрузки.

\section{1. Введение}

Испытания на ударный изгиб являются обязательными для большинства труб нефтегазового сортамента в качестве составляющей комплексного контроля механических свойств для обеспечения необходимого ресурса и безопасности эксплуатации. Особенно актуально данное испытание для труб, предназначенных для месторождений, расположенных в климатических районах с холодным климатом.

Применение для испытаний на ударный изгиб копров, оснащенных осциллографической записью диаграммы ударного разрушения в координатах усилие $F-n р о г и б ~ S$, дает возможность провести оценку различных стадий разрушений по этим параметрам [1-3]. При этом регистрируемый на всех стадиях разрушения образца прогиб $S$ связан с наложением двух одновременно протекающих процессов - изгибом образца при его макропластической деформации и раскрытием магистральной трещины $[1,3]$. В зависимости от ряда факторов вклад каждого из этих процессов может быть разным и определяется, в частности, механизмом разрушения: в случае хрупкого разрушения доля первого процесса мала, тогда как при вязком - существенна и должна учитываться.

Согласно стандарту ASTM E2298 - 13 [4], на осциллографической кривой выделяются характерные точки, соответствующие разным стадиям разрушения образца (рис. 1). Однако информация, заложенная в кривых $F-S$, далеко не ограничивается интерпретацией выделенных характерных точек. Совместный анализ осциллографических кривых с результатами фрактографических исследований позволяет получить более подробные сведения об особенностях процесса разрушения [5-7]. 


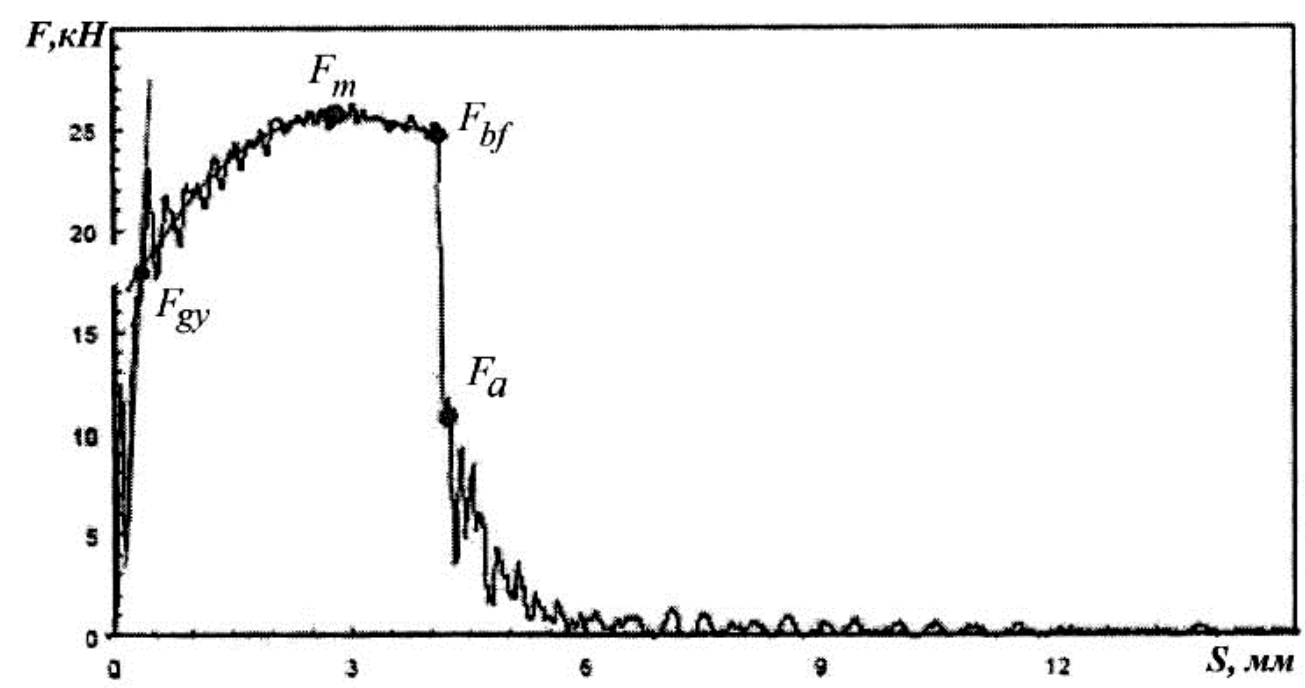

Рис. 1. Диаграмма нагрузка $F$ - прогиб $S$ при ударном деформировании образца Шарпи [4]

Цель настоящей работы - изучение механизмов разрушения низко- и среднеуглеродистых сталей на основе совместного анализа диаграмм ударного разрушения образцов Шарпи и их поверхностей разрушения.

\section{2. Материалы и методики исследования}

В качестве материала исследования использовали стали 05Г2СФ и 32Г2Р. Сталь 05Г2СФ применяется для изготовления сварных труб большого диаметра. Образцы были вырезаны из стенки труб диаметром 1420 мм с толщиной стенки 27,7 мм, сваренные из листа, полученного по режиму контролируемой прокатки с ускоренным охлаждением [8]. Образцы стали 32Г2Р вырезали из горячекатаных труб и подвергали нормализации от 1000 ㄷ. Микроструктура образцов после указанной термообработки представлена в [9].

Образцы Шарпи размером $10 \times 10$ мм разрушались при температурах $+20 \ldots-80{ }^{\circ} \mathrm{C}$ на копре с падающим грузом INSTRON CEAST 9350, оснащенным инструментальным бойком и системой сбора и обработки данных DAS Ceast 16000. Частота сбора данных составляла 1,0 МГц, что обеспечило интервал между двумя соседними точками 0,001 мс.

Первичная кривая ударного разрушения в координатах усилие $F-$ время $\tau$ для уменьшения колебаний системы опора - образец - молот программно (с помощью фильтра в системе DAS Ceast 16000) подвергалась фильтрации с частотой 2 кГц, а затем с помощью программы CeastView 5.94 двойному интегрированию для получения кривой в координатах усилие $F$ - прогиб $S$.

Затем на кривых $F=f(S)$ выделялись характерные точки, соответствующие: $F_{g y}$ - началу общей текучести, $F_{\max }$ - максимуму нагрузки, $F_{\min }$ - падению нагрузки между двумя максимумами, $F_{b f}$ - началу нестабильного хрупкого разрушения, $F_{a}$ - затуплению трещины при доломе, $F_{\kappa}$ - разрушению образца.

Фрактографический анализ разрушения включал выявление и оценку размеров зон в изломе образца Шарпи [10]:

- сдвига $\Theta$, примыкающей к надрезу,

- однородного вязкого разрушения $L_{C}$,

- волокнистого (вязкого) разрушения $L_{B}$,

- хрупкого разрушения $L_{X P}$,

- вязкого долома $L_{\text {д. }}$

Измерение величины зон $\left(\Theta, L_{B}, L_{C}, L_{X}, L_{\text {Д}}\right)$ с точностью 0,1 мм проводили на фотографиях изломов, полученных с пятикратным увеличением. 


\section{3. Результаты эксперимента и их обсуждение}

Диаграммы ударного разрушения высоковязких сталей типа 05Г2СФ с ультрадисперсной феррито-бейнитной структурой имеют ряд особенностей, которые не характерны для подобных диаграмм конструкционных сталей (рис. 2). Это расширяет наше представление о механизме вязкого разрушения и требует своей трактовки.
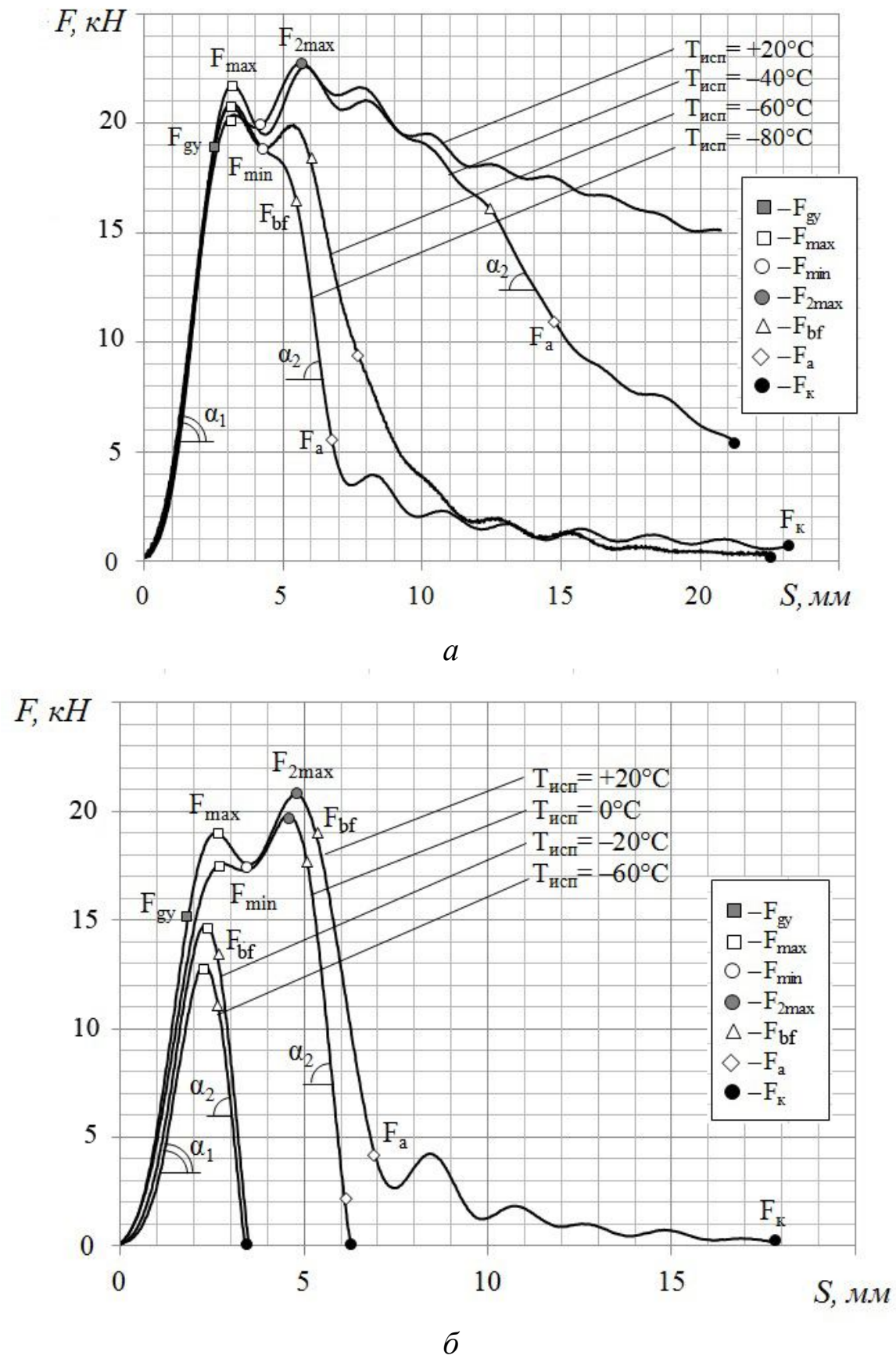

Рис. 2. Диаграммы разрушения образцов сталей 05Г2СФ (a) и 32Г2Р (б) при различных температурах испытания.

Полагаем, что до точки $F_{g y}$ происходит упругопластический изгиб образца, вероятно, ещё без формирования магистральной трещины. Из диаграммы на рис. 2 следует, что tg $\alpha_{1}$ не зависит от температуры испытания и примерно одинаков для обеих сталей. Это, очевидно, связано с тем, что в исследуемых сталях основной структурной составляющей, принимающей участие в пластическом течении, является феррит. 
Считаем, подобно [1-3], что на участке $F_{g y}-F_{\max }$ происходит зарождение магистральной трещины. Тогда на участках диаграммы $F-S$ после $F_{\max }$ происходит распространение магистральной трещины, сопровождающееся пластическим изгибом образца. Усилие, соответствующее началу роста магистральной трещины $\left(F_{\max }\right)$, в обеих сталях максимально при $T_{u c n}=20^{\circ} \mathrm{C}$ (для стали 05Г2СФ $P \approx 22 \kappa \mathrm{H}$, для стали $32 Г 2 \mathrm{P} P \approx 19 \mathrm{\kappa H}$ ) и с уменьшением температуры испытания $F_{\max }$ падает.

Как можно видеть на рис. 2 , кривая на участке $F_{g y}-F_{\max }$ понижается с уменьшением температуры испытания, так как зарождение магистральной трещины происходит при всё меньших нагрузках. Снижение кривой на участке $F_{\max }-F_{\min }$ связано с движением (страгиванием) магистральной трещины и релаксацией напряжений путем трещинообразования [11].

Для диаграмм разрушения $F-S$ обеих сталей характерно формирование второго максимума нагрузки $F_{2 \max }$, который для образцов, испытанных при повышенных температурах, даже выше первого $F_{\max }$. Рост сопротивления распространения вязкой трещины связан, по всей очевидности, с образованием в её окрестности области пластической деформации, где накапливаются экранирующие дислокации, поле напряжения которых противодействует напряжениям, приложенным к телу и вызывающим распространение трещины [3, 12].

Рисунок 2 показывает, что величина $F_{\max }$, характеризующая работу зарождения трещины, существенно снижается при понижении $T_{u c n}$ и/или при переходе от стали 05Г2СФ к стали 32Г2Р при близком значении прогиба образца $S$. Аналогичное, но более инертное поведение присуще точке $F_{\min }$.

Следовательно, зарождение трещины в обеих рассматриваемых сталях с ферритной матрицей происходит при близком значении макропластической деформации (прогибе образца), но различном уровне концентрации напряжений, поскольку с ростом вязкости стали макропластическая деформация релаксирует всё большую часть упругой энергии, концентрация которой на надрезе образца требуется для зарождения и страгивания трещины.

С понижением $T_{u c n}$ и переходе от стали 05Г2СФ к стали 32Г2Р $F_{2 \max }$ не только активно уменьшается по величине, но смещается в сторону меньших $S$ и при низких $T_{u c n}$ вообще исчезает. Изменение $K C V, F_{\max }$ и $F_{2 \max }$ с понижением температуры испытаний у образцов обеих сталей представлено на рис. 3 . В области полностью вязкого разрушения $F_{2 \text { max }}$ коррелирует с $K C V$ для обеих сталей. $F_{\max }$ отражает напряжение необходимое для зарождения и страгивания трещины, и его величина у стали $32 \Gamma 2 \mathrm{P}$ заметно падает в области ниже $T_{X P}$ при $T_{u c n}=-20 ;-60^{\circ} \mathrm{C}$.

После $F_{2 \max }$ (или $F_{\max }$ ) в зависимости от уровня вязкости сталей наблюдается различный ход кривых на диаграммах разрушения $F=f(S)$ (см. рис. 2):

1) осциллирование нагрузки $F$, особенно ярко выраженное для сталей в высоковязком состоянии (сталь $05 Г 2 \mathrm{CФ}, T_{u c n}=20^{\circ} \mathrm{C}$ ), завершающееся остановкой трещин в недоломанных образцах;

2) резкое снижение кривой по гладкой монотонной траектории, что характерно для хрупко разрушенных образцов (сталь $32 \Gamma 2 \mathrm{P}, T_{u c n}=-20 ;-60{ }^{\circ} \mathrm{C}$ );

3 ) сочетание этих двух типов кривых, когда осциллирующий участок в $F_{b f}$ вплоть до $F_{a}$ сменяется гладкой ниспадающей кривой (сталь $05 Г 2 \mathrm{C} \Phi, T_{u c n}=-40{ }^{\circ} \mathrm{C}$ ).

Наибольшее распространение имеет третий тип кривых $F=f(S)$, когда разрушение образца происходит по смешанному механизму с различной долей вязкой и хрупкой компонент, что задает на диаграммах положение $F_{b f}$ и $F_{a}$. С понижением уровня вязкости стали $F_{b f}$ и $F_{a}$ закономерно смещаются в сторону меньших значений $F$ и $S$, что приводит увеличению угла $\alpha_{2}\left(\operatorname{tg} \alpha_{2}\right)$ (см. рис. 2,3$)$.

Это совпадает с подходом в стандарте ASTM E2298-13 [4]: в $F_{b f}$ появляется компонента хрупкого разрушения металла. 


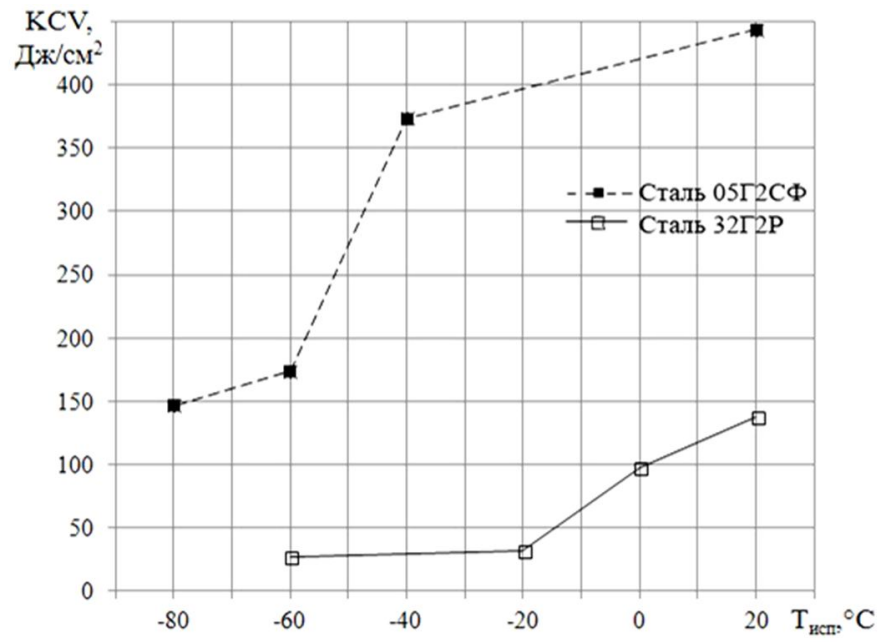

$a$

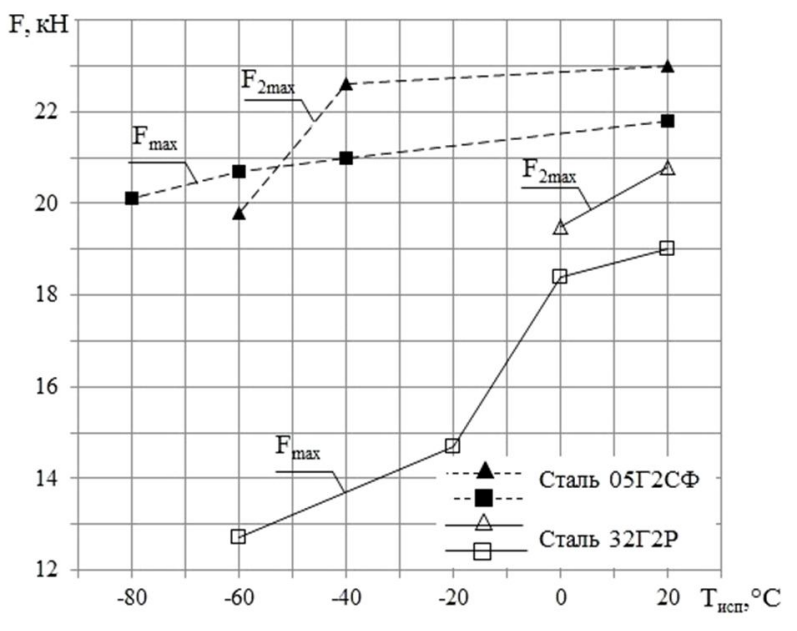

6

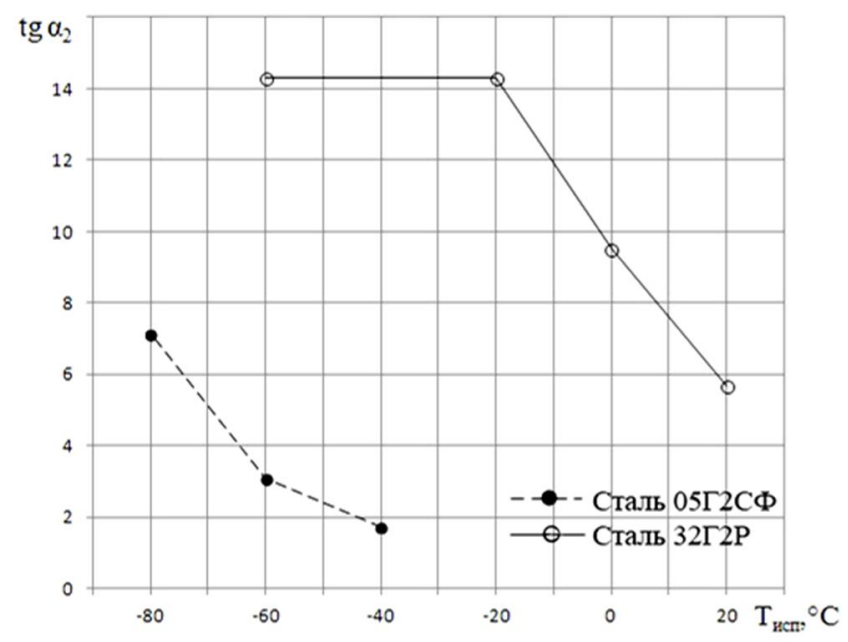

B

Рис. 3. Сериальные кривые ударной вязкости исследованных сталей $(a)$ и изменение величин максимумов $\left(F_{\max }, F_{2 \max }\right)$ (б) и $\operatorname{tg} \alpha_{2}\left(\right.$ (в) с понижением $\mathrm{T}_{\text {исп }}$

Итак, наиболее полная информация о вязкости стали формируется при одновременном использовании обоих критериев: $F_{\max }\left(F_{2 \max }\right)$ и $\operatorname{tg} \alpha_{2}$ как наиболее чувствительных и однозначно характеризующих её склонность к зарождению и распространению трещины. Кстати, из анализа данных критериев наглядно видна крайне высокая трещиностойкость стали 05Г2СФ с феррито-бейнитной (мартенситной) структурой и сверхмелким зерном, полученными по технологии контролируемой прокатки с ускоренным охлаждением.

На завершающем этапе, после $F_{a}$, может вновь появиться биение кривой $F=f(S)$, что является индикатором возникновения вязкой компоненты разрушения образца при доломе (рис. 2). В крайне охрупченном состоянии (сталь $32 \Gamma 2 \mathrm{P}, T_{u c n}=-20$ и $-60^{\circ} \mathrm{C}$ ) этот эффект отсутствует.

Привязка стадий разрушения к зонам на поверхности изломов образиов. «Критические» точки (точки перелома, экстремумов) на кривых $F=f(S)$ показывают смену величины и темпа падения разрушающей и деформирующей образец нагрузки по мере изгиба образца, а следовательно, смену механизмов разрушения. Несомненно, они имеют адекватное отражение на поверхности изломов образцов, так как каждый механизм распространения трещи- 
ны проявляется как свой характерный набор параметров рельефа поверхности разрушения $[10,13]$.

Участок $F_{g y}-F_{m}\left(F_{\max }\right)$, по всей вероятности, соответствует зоне $\theta$, где происходит зарождение и страгивание трещины. На недоломанных образцах стали $05 Г 2 С \Phi\left(T_{u c n}=20{ }^{\circ} \mathrm{C}\right)$ трещина успевает пройти до своей остановки, в основном зону однородного вязкого разрушения $L_{C}$, что сопровождается биением нагрузки $F$. Наступление стадии совместного действия вязкого и хрупкого механизмов распространения трещины длится от $F_{\max }$ вплоть до $F_{a}$. Соответственно участок $F_{b f}-F_{a}$, где, очевидно, действие охрупчивающей компоненты в стали 05Г2СФ проявляется как зона $L_{B}$, в которой появляются расщепления при общем макровязком механизме разрушения [11], а в стали 32Г2Р - как область хрупкого квадрата (рис. 4).
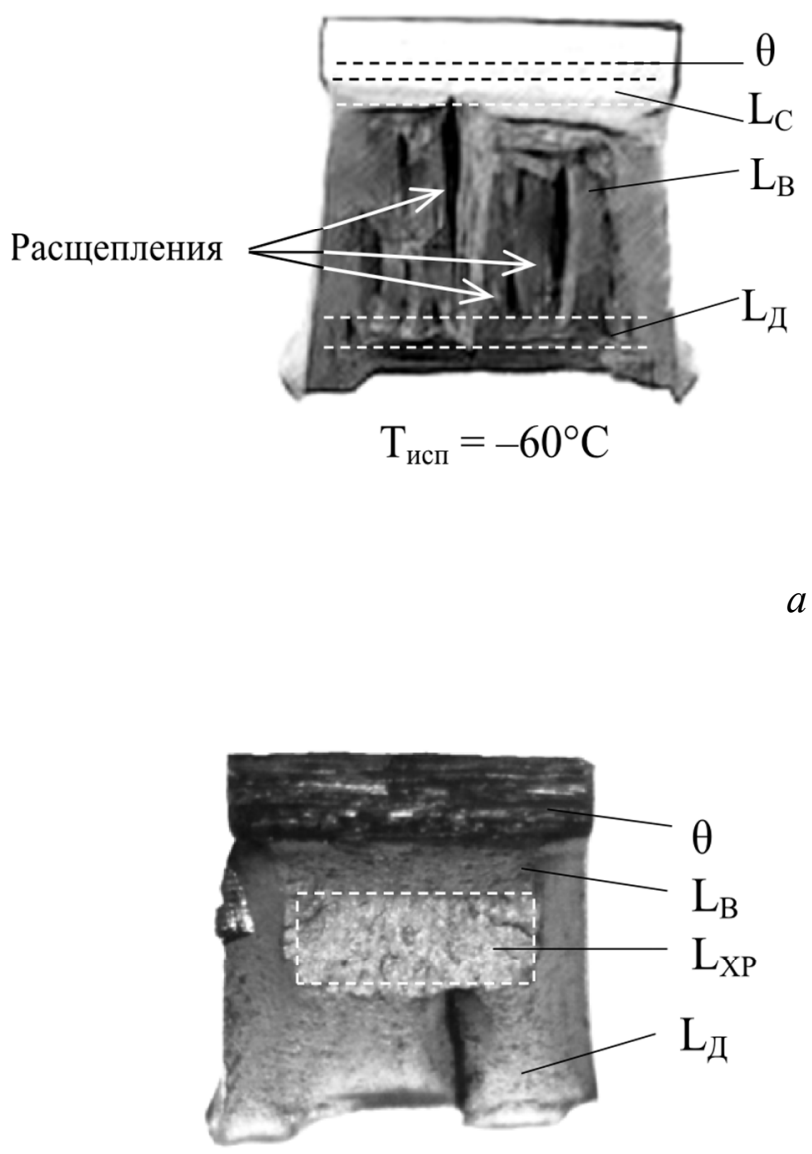

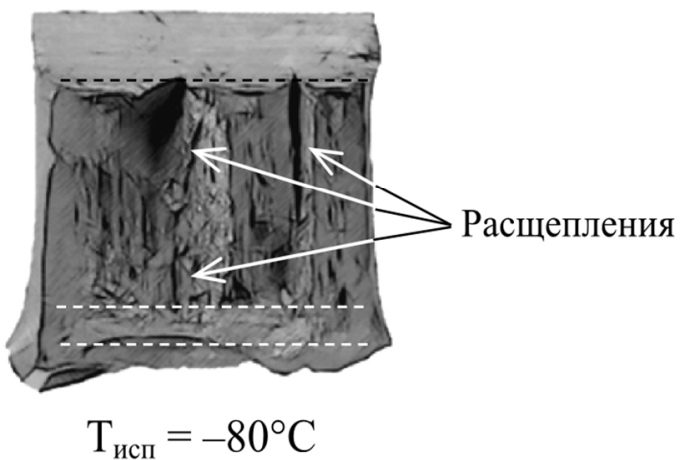

$a$

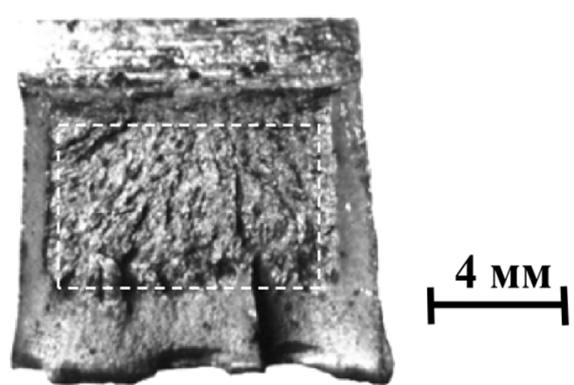

Рис. 4. Поверхности разрушения образцов Шарпи сталей 05Г2СФ (a) и 32Г2Р (б) после испытаний при различных температурах

Зона вязкого разрушения при доломе (зона $L_{Д}$ ) проявляется как возобновление биения кривой $F=f(S)$, что при доминирующем хрупком разрушении образца (сталь $32 \Gamma 2 \mathrm{P})$ не регистрируется.

\section{4. Выводы}

1. Совместным анализом отфильтрованных диаграмм разрушения в координатах нагрузка $F$ - прогиб $S$ при ударном изгибе образцов Шарпи сталей 05Г2СФ и 32Г2Р, сери- 
альных кривых ударной вязкости и поверхности разрушения установлено, что при максимально вязком механизме распространения трещины происходит осцилляция кривых $F-S$ при весьма замедленном их снижении как на стадии зарождения, так и на стадии распространения вязкой трещины

2. Хрупкому или хрупко-вязкому механизмам распространения трещины соответствует сглаженный монотонно снижающийся участок кривой $F-S$, тангенс угла которого к оси абсцисс коррелирует с ударной вязкостью $K C V$.

3. Устновено, что каждому участку на кривой $F-S$ между точками перегиба (экстремума) соответствует своя зона на поверхности излома образцов Шарпи: зоны чистого вязкого разрушения $\left(\theta+L_{C}\right)$ проявляются как осциллирующий участок кривой на диаграмме $F-S$; сглаженный снижающийся участок $F-S$ обусловлен появлением хрупкой компонентой разрушения, связанной в стали 05Г2СФ с появлением зоны $L_{B}$ и расщеплений в ней, в стали 32Г2Р - хрупкого квадрата (зона $\left.L_{X P}\right)$.

\section{Литература}

1. Гудков А. А. Трещиностойкость стали. - М. : Металлургия, 1989. - 376 с.

2. Комплексный анализ механических свойств материала для обоснования ресурса и безопасности продуктов / М. А. Махутов, В. Б. Пермяков, Л. Р. Ботвина, Ю. А. Кравцова // Проблемы безопасности и чрезвычайных ситуаций. - 2006. - № 3. - С. 65-76.

3. Ботвина Л. Р. Разрушение: кинетика, механизмы, общие закономерности. M. : Наука, 2008. - 334 с.

4. ASTM E2298. Standard test method for instrumented impact testing of metallic materials // ASTM. - 2013. -9 p.

5. Manahan M. P., Stonesifer R. B. The difference between total absorbed energy measured using an instrumented striker and that obtained using an optical decoder // in book «Pendulum Impact Testing: A Century of Progress». - ESIS STP 1380. - 2000. - P.181-197.

6. Tronskar J. P., Mannan M. A., Lai M. O. Measurements of fracture initiation toughness and crack resistance in instrumented Charpy impact testing // Eng. Fract. Mech. - 2002. - Vol. 69. P. 321-338.

7. Viehrig H.-W., Boehmert J., Richter H., Valo M. Use of instrumented Charpy test for determination of crack initiation toughness // in book «Pendulum Impact Testing: A Century of Progress». - ESIS STP 1380. - 2000. - P. 354-365.

8. Микроструктура и дисперсные фазы в высокопрочных сталях газопроводных труб большого диаметра / А. Б. Арабей, В. М. Фарбер, И. Ю. Пышминцев, А. Г. Глебов, О. В. Селиванова, Н. В. Лежнин // Наука и техника в газовой промышленности. - 2011. № 4. - С. 86-91.

9. Изыскание составов и режимов термической обработки обсадных и насоснокомпрессорных труб высокой прочности / Н. Т. Тихонцева, П. Ю. Горожанин, С. Ю. Жукова, М. Н. Лефлер, В. М. Фарбер // Сталь. - 2006. - № 8. - С. 70-73.

10. Фрактографическая диагностика трещиностойкости труб группы прочности X80 (К65) по результатам испытаний ударной вязкости / В. М. Фарбер, А. Б. Арабей, И. Ю. Пышминцев, В. А. Хотинов, А. Н. Журавкова, Е. Н. Чусова // XVIII Межд. науч.-техн. конф. «Трубы2010» : материалы науч. конф. - Челябинск : РосНИТИ, 2010. - С. 108-117.

11. Фарбер В. М., Селиванова О. В. Классификация процессов релаксации напряжений и их проявлений при пластической деформации металлов // Металлы. - 2001. - № 1. С. $110-114$.

12. Кан Р. У., Хаазен П. Физическое металловедение: В 3 т. - М. : Металлургия, 1987. T. 3. -664 c. 
opent-aceess journal

Issue 2, 2015

13. Особенности разрушения трубных сталей класса прочности Х80 (К65) / А. Б. Арабей, И. Ю. Пышминцев, В. М. Фарбер, В. А. Хотинов, А. О. Струин // Известия ВУЗов. Черная металлургия. - 2012. - № 3. - С. 12-20. 